\title{
Generalised quantifiers and the semantics of the same*
}

\author{
R. Zuber \\ CNRS, Paris
}

\begin{abstract}
The notions of the nominative case extension of a type $\langle 1\rangle$ quantifier and of the dual of type $\langle 1\rangle$ quantifier are used to present the semantics of various, structurally different, expressions containing the same.
\end{abstract}

Keywords: Case extensions of quantifiers, dual quantifiers, generalised conservativity

\section{Introduction}

It is quite natural that the formative same, several of its compounds and a similar formative like different have given rise to intensive research and numerous publications or even important unpublished work (see Barker 2007 for an overview of an important part of the relevant literature on the subject). The reasons for interest in those items are both syntactic and logico-semantic. For instance, one can quickly realise that their syntactic status cannot be easily determined and, moreover, that they are categorially polyvalent. More importantly, these items are of considerable logical interest since their semantics goes immediately beyond standard first order analysis and they give rise to specific less well-known inference patterns. In this paper I am basically interested in logical and methodological aspects suggested by the semantic analysis of these formatives. In particular I will show how generalised quantifier theory enriched by case extension of quantifiers can be applied to their semantics.

Two remarks about the data to be discussed. First, I will not directly discuss sentences in which a complete interpretation of the same is impossible without making reference to some "sentence-external" (linguistic) elements in order to take into account the "other part" of equality (or comparison) induced by the same. An example of such a possibility is given by the sequence in (1) which to be fully interpreted necessitates an explicit reference to elements not occurring in the sentence itself as in (2a) or (2b):

(1) Leo is reading the same book.

(2) a. Leo is reading the same book as yesterday.

* A couple of thanks to Ross Charnock for comparing his English to mine. I hesitate to thank David. 
b. Leo is reading the same book as Lea.

Sentences like (1) will be called sentences in standard form, and sentences similar to those in (2), that is, roughly speaking, sentences with the same and the complementizer as, will be called sentences in the comparative form. Though I will not be interested in deictic uses of the same (that is sentences with sentence-external readings of the same), I will discuss some sentences in the comparative form and their relationship with some constructions containing the same and which are not in the comparative form.

Observe that sentences in which the same is used twice, for instance in subject and object positions, must be interpreted deictically with the help of sentenceexternal elements (when taken in non-generic reading). This is the case with (3a), for instance, which, it seems to me, can be interpreted only by sentences in comparative form as in (3b):

(3) a. The same students ordered the same dishes.

b. The same students ordered the same dishes (that they ordered) last week.

I will thus be interested basically in sentences containing the same in object position and which can be interpreted sentence-internally, that is without explicit reference to elements not present in the sentence as (4):

(4) The five students read the same book.

Observe that a sentence-internal reading is an additional reading of sentences with the same: (4) still has a sentence-external reading which can be represented by a sentence in comparative form. Moreover, there are sentences, like (1) above, and possibly (3a) and (3b), that can have only sentence-external readings.

The second point concerning the data has, roughly speaking, to do with what philosophers of language would call the type-token distinction. Obviously the same involves some kind of identity between objects and thus when interpreting sentences with the same a question arises as to just how similar two objects have to be in order to count as the same. In (4) for instance both identities, type identity and token identity, are possible: the students could have read the same token of the book or the same "type", roughly speaking, potentially including even just a translation of some book. Observe that this example shows that type-token distinction is not really appropriate, and probably, as shown in Lasersohn 2000, the degree of similarity matters. The problem is linguistically important since the situation can depend on the verb used: dating the same woman or climbing the same hill strongly imply token identity whereas eating the same dish or drinking the same water involves type identity. In addition the type of identity required may depend on the tense and aspect used in the verb. In fact, some languages have different lexical items allowing type 
identity and token identity to be distinguished. This is probably the case in Polish with taki sam and ten sam or in German with der gleiche and derselbe.

\section{Formal Preliminaries}

Let me start by recalling some basic notions from the "classical" generalised quantifier theory and some less classical extensions and generalisations which will be used in the sequel. As we will see, these extensions basically have a linguistic justification.

Given a fixed universe $E$, a type $n$ quantifier is a function from $n$-ary relations to sets. If $n>1$ then such functions are called polyadic quantifiers. A type $\langle 1\rangle$ quantifier is a function from sets (subsets of $E$ ) to truth values, and thus it is a set of subsets of $E$. A $\langle 1\rangle$ quantifier $Q$ will be called plural iff any of its members contains at least two elements. A type $\langle 1,1\rangle$ quantifier is a function from sets to type $\langle 1\rangle$ quantifiers. Type $\langle 1\rangle$ quantifiers are denotations of noun phrases and (a class of a) type $\langle 1,1\rangle$ quantifiers are denotations of (unary nominal) determiners, that is expressions like every, no, most, five, etc. Since both types of quantifiers form Boolean algebras, they have Boolean complements (negations): if Q is a quantifier (of one of the above indicated types) then $\neg Q$ is its Boolean complement. Both types of quantifiers also have post-negations: if $Q$ is a type $\langle 1\rangle$ quantifier then its postnegation $Q \neg$ is defined as: $Q \neg=\left\{X: X^{\prime} \in Q\right\}$, where $X^{\prime}$ is the Boolean complement of $X$. If $Q$ is a type $\langle 1,1\rangle$ quantifier then $Q \neg$ is that type $\langle 1,1\rangle$ quantifier which for every set $X$ associates to $Q(X)$ the post-complement of $Q(X): Q \neg(X)=Q(X) \neg$.

Both types of negations can be used to define the dual quantifier $Q^{d}$ of a given quantifier $Q$ : by definition $Q^{d}=\neg(Q \neg)=(\neg Q) \neg$. Obviously dual quantifiers are symmetric in the sense that $\left(Q^{d}\right)^{d}=Q$. For instance $E V E R Y$ and $N O$ (considered as type $\langle 1,1\rangle$ ) are dual of each other. A quantifier which is equal to its dual is called self-dual. Usually one considers proper nouns to denote ultrafilters generated by the individuals referred to by the proper noun: if $\operatorname{Pr} N$ refers to the individual $a$ then $\operatorname{Pr} N$ denotes the quantifier $I_{a}=\{X: a \in X\}$ (where $X \subseteq E$ ). It is easy to check that quantifiers denoted by proper nouns are self-dual. Dual and self-dual quantifiers are discussed by Keenan (2005), who is interested in specific inference patterns to which they give rise. The application of the notion of the dual quantifier will be given in the next sections, where the description of the semantics of sentences with constituents containing the same will be proposed.

The above notions belong to the "classical" generalised quantifier theory. This means that basically type $\langle 1\rangle$ quantifiers are functions from sets to truth values. Linguistically such functions interpret noun phrases in (grammatical) subject positions. However, noun phrases can occur not only in subject position and thus, at least for linguistic reasons, their domain of application should be extended so that they also 
apply to $n$-ary relations.

To see this more precisely consider examples in (5) and (6):

(5) Five logicians whistled.

(6) Two philosophers called five logicians.

In (5) the quantifier FIVE-LOGICIANS applies to the property WHISTLE and gives truth or falsity depending on whether this property belongs to the quantifier or not. Concerning (6), when it is interpreted with subject-NP wide scope, there are syntactic reasons in this case to consider the noun phrase five logicians to apply to the transitive verb call(ed) and give the verb phrase called five logicians. Since transitive verbs are interpreted by binary relations this means that the type $\langle 1\rangle$ quantifier FIVE-LOGICIANS applies to the binary relation CALL and gives a property CALLFIVE-LOGICIANS (to which the quantifier TWO-PHILOSOPHERS applies).

Of course noun phrases can also occur in other positions than subject or direct object positions. This means that the domain of application of type $\langle 1\rangle$ quantifiers should be extended so that they apply also to $n$-ary relations. Here we will consider only a particular case of extension to binary relations (supposed to be denotations of transitive verbs). A type $\langle 1\rangle$ quantifier $Q$ can apply in two ways to binary relations: as a "subject function", called its nominative extension $Q_{\text {nom }}$, and as a "direct object function" called the accusative extension $Q_{a c c}$. Both of these domain extensions are case extensions (of a type $\langle 1\rangle$ quantifier).

Case extensions are formally defined in the following way. Let $Q$ be a type $\langle 1\rangle$ quantifier, $R$ a binary relation and $a \in E$. Then $a R=\{x:\langle a, x\rangle \in R\}$ and $R a=\{x:\langle x, a\rangle \in R\}$. Given this notation $Q_{\text {nom }}$ and $Q_{a c c}$ are defined as follows: $Q_{n o m}(R)=\{x: Q(R x)=1\}$ and $Q_{a c c}(R)=\{x: Q(x R)=1\}$. If it is clear that $R$ is a binary relation, $Q_{\text {nom }}(R)$ will be noted $Q(R)$ from now on. We see that the value of a case extension of the quantifier $Q$ at a binary relation is uniquely determined by the value of $Q$ at a set.

It follows from the above definitions that if $Q=$ FIVE-LOGICIANS and $R=$ $C A L L$ then $Q_{\text {nom }}(C A L L)$ is the set of all objects to which five logicians are in the relation of calling and $Q_{a c c}(C A L L)$ is the set of all objects which are in the relation of calling to five logicians.

Quantifiers are specific functions having Boolean structure, that is functions forming (pointwise) a Boolean algebra and mapping a Boolean algebra into a Boolean algebra. Case extensions are this type of function. They can be considered as arity reducers by 1: when applied to a binary relation they give a set as result, that is a unary relation. More generally, extensions of type $\langle 1\rangle$ quantifiers are arity reducers by 1 . For instance, in the more general case, when oblique NPs are considered with ditransitive verbs, the quantifiers denoted by such oblique objects reduce ternary relations to binary ones. 
The notion of post-negation and of a dual generalises to functions having a Boolean structure. Thus if $F$ has a Boolean structure then $F \neg(X)$, the post-negation of $F$, is defined as $F \neg(X)=_{\text {def. }} F\left(X^{\prime}\right)$. Similarly $F^{d}(X)$, the dual of $F$, is defined as $F^{d}(X)=_{\text {def. }} \neg F \neg(X)$. Case extensions have Boolean structure.

Given the above definitions it is easy to show the following dependency for postnegations and duals of case extensions of a given quantifier $Q:\left(Q_{\text {nom }}\right) \neg=(Q \neg)_{\text {nom }}$ and $\left(Q_{\text {nom }}\right)^{d}=\left(Q^{d}\right)_{\text {nom }}$. Similarly for the accusative case extension.

In the following sections an application of the notions of the dual quantifier and of the nominative case extension to the semantics of various types of sentences with the same will be given.

\section{Application of dual quantifiers}

As we have seen, noun phrases with the same have many uses. In order to show how the notions of the case extension and of the dual quantifier can be applied to the semantics of the same, I start with simple constructions like the one in (7):

(7) Every student read the same books.

I will be basically interested in the logical or non-presuppositional semantic interpretation of such constructions. On this interpretation of (7) it is not presupposed that any reading of any book by any student took place. Thus on this reading (7) is true if no student read any book. Similarly the semantic impact of the plural in the object NP will be ignored for the moment. Observe for instance that (8a) and (8b) do not sound contradictory and consequently such a reading is not absurd:

(8) a. Every student read the same book, namely none.

b. Every student answered the same question, namely none.

It has been established (Keenan 1992) that in such constructions so-called Fregeirreducible type $\langle 2\rangle$ quantifiers are involved. More precisely, if $R$ is the binary relation corresponding to reading, then for no two type $\langle 1\rangle$ quantifiers $Q$ and $Q_{1}$ can the semantics of (7) be represented in the form $Q_{1}\left(Q_{a c c}(R)\right)$. Thus in (7) we have a polyadic quantifier which binds more than one variable ("at the same time"). Observe in addition that syntactically the noun-phrase-like expression the same books cannot be considered a true noun phrase since it cannot occur as grammatical subject in sentences with an intransitive verb. Moreover, one cannot say that it denotes the accusative extension of a type $\langle 1\rangle$ quantifier.

It is important to keep in mind that Keenan's result presupposes that irreducible quantifiers are denotations of a peculiar "constituent" composed of a sequence of a specific NP in subject position and of the verbal argument with the same in the object position. 
What (7) essentially says is that no book is such that it was read by some but not by all students, or equivalently that any book that was read by any student was read by every student. Thus (7) should satisfy condition given in (9a) or (9b):

$$
\begin{aligned}
& \text { a. } N O(B)[(S O M E(S) \wedge \neg E V E R Y(S)] R=1 \\
& \text { b. } S O M E(S) R \cap B \subseteq E V E R Y(S) R \cap B
\end{aligned}
$$

Sentence (7) is just a particular case of constructions with the same occurring in the direct object NP. An important point is that in (7) the quantified noun phrase in the subject position can be replaced by many other quantified noun phrases and even non-quantified ones. This can be seen in (10):

(10) No students / most students / five students / at least two students / Leo and Lea read the same book(s).

It is not easy to describe restrictions on noun phrases in sentences of the above type. It seems clear that semantically they should be, roughly speaking, plurals. For instance the interpretation of (4) should be the same as the interpretation of (11), and (12a) should have the same interpretation as (12b):

(11) Every two students read the same book.

(12) a. No students read the same book.

b. No two students read the same book.

The above examples show that the NPs in question should probably denote plural quantifiers. There are, however, other restrictions:

(13) a. Every student except Leo answered the same question.

b. *No student except Leo answered the same question.

There is a difference in the grammaticality of (13a) and (13b) which is not simple to analyze. I will not be concerned with this problem any further, however, and still consider there to be a need to make some generalisations in order to obtain a formula similar to the one in (9) which would account for the semantics of sentences like those in (10). Such sentences can be said to be of the linguistic form given in (14):

NP TV THE-SAME(X), where TV is a transitive verb.

As indicated above the NP in (14) can denote not only EVERY but many other type $\langle 1\rangle$ quantifiers.

To solve the more general problem posed by the semantics of (14), recall that $S O M E$ is the dual of EVERY. So the move I propose is to generalise the conditions in $(9 a)$ or $(9 b)$ and to consider sentences of the form in (14) to have the semantics given in (15): 
$Q^{d} R \cap X \subseteq Q R \cap X$, where $\mathrm{Q}$ is the denotation of NP and $\mathrm{R}$ - the denotation of TV.

Let us see one consequence of (15), which can be considered as its indirect justification. Observe that if (7) is true, then (the set of) the books which were not read by all students is also the same (for each student) and it is the complement of the set of the books which were read by all students. This means that the interpreting function is constant on complements: it takes the same value for relation $R$ and its complement $R^{\prime}$. More precisely, using the definition of dual quantifiers and simple set-theoretical equivalences, one shows that (15) holds iff (16) holds:

$$
Q^{d} R^{\prime} \cap X \subseteq Q R^{\prime} \cap X
$$

Let me illustrate the proposal made in (15) with three additional examples. First, consider the simplest example given in (17):

(17) Leo and Lea read the same books.

The dual of the quantifier denoted by the conjunction Leo and Lea is the quantifier denoted by the disjunction Leo or Lea. Thus, according to (15), the sentence in (17) means (on its non-presuppositional reading) that there is no book which has been read by either Leo or by Lea but not read by Leo and Lea. This statement is indeed equivalent to the truth-conditions of (17).

As another illustration, consider now a slightly more complicated case with most in subject position. The dual of MOST is AT-LEAST-HALF. Condition (15) says that sentence (18) is true when and only when every book which has been read by half of the students has been read by most of them or, equivalently, no book is such that it was read by half of students and not by the majority of them:

Most students read the same books.

The above characterisation of (18) looks reasonable and can be easily checked as correct, at least when the universe $E$ is very limited in size.

Finally consider the more complicated example given in (19a). I consider (19a) to be equivalent to (19b) and thus that to be true if and only if (19c) is false:

a. No three students read the same book.

b. It is not true that three students read the same books.

c. Three students read the same books.

In (19a) the NP no three students denotes the the quantifier $\neg 3(S)$, where $3(S)(Y)=1$ iff $|S \cap Y| \geq 3$. The dual of this quantifier is the quantifier $3(S) \neg$. Consequently, given the pattern in (15), the semantics of (19a) is given in (20): 


$$
B \cap \neg 3(S)(R) \nsubseteq B \cap 3(S) \neg
$$

Consider now a related example in (21a). As said above, it can be considered to be equivalent to (21b), if you accept (21b). Their semantics is given in (22):

(21) a. No two students read the same book.

b. No students read the same book.

$$
B \cap \neg 2(S)(R) \nsubseteq B \cap 2(S) \neg
$$

Thus one cannot apply the formula (15) in the case of (21b) because strictly speaking we do not have either the quantifier $N O(S)$ nor its Boolean complement in subject position in (21b).

To conclude this series of examples, I just indicate an example of sentences with the same in predicative position, or sentences in the predicative form:

The books that most students read are the same.

The fact that such sentences contain a relative clause implies more restrictions on the NPs which can occur in them. For instance, there is no predicative form corresponding to (21a). Sentences in predicative form are usually semantically equivalent to corresponding sentences in the standard form, with possible additional presuppositions due to the relative clause they contain. Consequently, one can consider (23) to have the same truth conditions as (18).

In all the above examples the same denotes a function which is fixed by postnegation: it takes the same value for a relation and its complement, as was argued for (7). For instance if (18) is true then there is a set of books read by the majority of students. But at the same time the complement of the above set of books is the set of books not read by (the same) majority of students, and thus most students have "not read" the same books. In the next section I consider some examples for which this property does not hold.

\section{Other examples}

The semantics of the examples discussed in the previous section involves a function $F$ from sets and binary relations which takes the same value on a given relation and its complement. This property does not hold for other examples. To see this, consider first as a particular case constructions in which the formative the same is followed by a numeral, as in (24). Observe that (24) does not entail (7) because of (25):

(24) Every student read the same $n$ books. 
(25) Every student read the same $n$ books and $m$ different ones.

Sentence (24) is probably ambiguous, one reading entailing that every student read $n$ different tokens of the same (type) book. Here I am not interested in such a reading.

Constructions involving numerals are often ambiguous. So probably (24) has two readings, the "exactly reading" whose general form is given in (26a), and the "at least reading" indicated in $(26 \mathrm{~b})$ :
a. $|Q R \cap B|=n$
b. $|Q R \cap B| \leq n$

What (27a) and (27b) say is that (24) is equivalent to the object wide-scope reading of (27):

\section{(27) Every student read $n$ books.}

Let us see now briefly sentences of the form given in (28), that is sentences in comparative form. Instances of such sentences are given in (29) and (30):

$N P_{1} T V P T H E-S A M E(C N)$ as $N P_{2}$

Leo read the same books as Lea.

(30) Most students read the same books as every teacher.

In (28) the $N P_{1}$ and $N P_{2}$ can be virtually any NPs, in particular they can be singular NPs. When both $N P_{1}$ and $N P_{2}$ are plural an ambiguity can arise depending on whether the subject NP is taken in distributive or non-distributive reading.

The following fact is important for the semantics of sentences in the comparative form: (29) is not equivalent to (31). The reason is that we have sentences like (32). Consequently (28) is equivalent to the conjunction given in (33):

(31) Leo and Lea read the same books.

(32) Leo read the same books as Lea and (even, in addition) five more.

$$
N P_{1} T V P T H E-S A M E(C N) \text { as } N P_{2} \text { and } N P_{2} \text { TVP THE-SAME }(C N) \text { as } N P_{1}
$$

It follows from the above remarks that sentences in the comparative form given in (28) have truth conditions indicated in (34) and the conjunction in (33) obviously has the truth-conditions indicated in (35):

$$
\begin{aligned}
& Q_{2} R \cap X \subseteq Q_{1} \cap X \\
& Q_{1} R \cap X=Q_{2} \cap X
\end{aligned}
$$


The formulae in (15) and (35) indicate how to associate, at least in theory, a sentence in the standard form with a sentence in comparative form. Such an association implies that one can associate a plural $N P_{1}$ denoting the quantifier $Q$ with an $\mathrm{NP}_{2}$ denoting the dual of $Q$. This is not always possible.

To conclude this section I want to consider a sub-class of examples involving multiple events due to conjunctions of verb phrases. As Carlson (1987) points out, the sentence-internal reading depends on distributing over separate events and a comparison of them. In the sentences discussed until now a comparison of events due to actions performed by at least two agents is involved. For this reason, the subjects of such sentences must be plural. Multiple events can be also obtained in the case of conjunction of verb phrases or quantificational adverbs. In such cases, one agent performs actions giving rise to multiple events which are compared or differentiated. This is the case in the following examples:

(36) Leo bought and read the same books.

(37) Leo read the same book three times.

Example (37) involves a quantificational adverb whose semantic description is outside the scope of this paper.

Sentence (36) is an instance of the sentence in conjunctive form given in (38). Of course its verb phrase can contain more than two conjuncts. Using the notion of nominative extension of a quantifier we get the semantics of such sentences in (39):

$$
N P T V P_{1} A N D T V P_{2} T H E S A M E(C N)
$$

$$
Q R_{1} \cap X=Q R_{2} \cap X
$$

In conjunctive sentences, numerical modification can also take place. An example of such a sentence is given in (40) and its semantics is given in (41):

(40) Leo sold and read the same five books.

(41) $\left|I_{L} S \cap I_{L} R \cap B\right|=5$, where $I_{L}$ is the denotation of Leo, $R$ is the denotation of read, and $S$ the denotation of sell.

Given that plurality of events can be introduced either by plural subjects or by conjunctions of verb phrases, sentences where both occur are ambiguous:

(42) Leo and Lea bought and read the same books.

On the multiple events reading, sentence (42) can be true even if Leo read and bought different books than Lea. 
Semantics of the same

\section{The same and different}

There are many other formatives which are similar to the same semantically and from the point of view of their syntactic category. A great deal of the literature is in particular devoted to the formative different and its relationship with the same. In this section I will briefly discuss the semantics of different when it occurs in constructions similar to those in which the same occurs. Many problems will however be left aside since, interestingly, the semantics of different looks more complicated than the semantics of the same. A short discussion concerning the grammatical category of these formatives and of the logical type of their denotations will be given in the next section. I will also show that the functions they denote have the property of generalised conservativity.

One of the first things to be noted concerning different is that it can occur in quite similar types of constructions to the same. Similarly it gives rise to sentence-external (deictic) and sentence-internal interpretations and can thus occur in sentences in the comparative form. Moreover, as it is the case with the same, when different occurs in sentences whose subject NP denotes a non-plural quantifier then only the sentence-external interpretation is possible. These facts are instantiated in the following examples:

(43) Every student read a different book.

(44) Every student read a different book from the one that Lea read.

(45) Lea read Exciting Humor. Leo read a different book.

(46) The books that Leo and Lea read are different.

(47) Leo read different books than/*as Lea did.

Sentence (43) has the standard form. It can have a sentence-external interpretation, for instance the one in (44) or in (45), and a sentence-internal interpretation. In (44) and (47) we have a comparative form of the sentence with different and in (46) we have a predicative form. Notice that in the case of the comparative form the complementizer than is preferable.

One notices furthermore that different can also occur with numerals, as is the case with the same: the sentence in (48) is similar to the one in (24) above. In addition (48) does not entail (43) because of (25):

(48) Every student read $n$ different books.

Finally, different can occur in conjunctive sentences, that is sentences with conjoined verb phrases which involve multiple events:

Leo bought and read different books. 
We can now consider some semantic properties of different. Consider first a sentence in the standard form given in (50):

(50) Leo and Lea read different books.

One should notice incidentally that the verbal argument containing different and which plays the role of the direct object should be in the plural, in opposition to the case with the same: (51) does not seem to be well-formed (on the sentence-internal reading). Instead (52) should be used:

*Leo and Lea read a different book.

(52) Leo and Lea did not read the same book.

Sentences with different, as well as those with the same, indicate, roughly speaking, difference (or identity) between sets. Formally two sets are different when there is an element which belongs to one set but not to the other one. However, one can also think about a stronger difference between sets: two sets are strongly different when they do not have a common element. So the question one might ask is which difference applies to the semantics of sentences in which different occurs. If, roughly, different is equal to not the same, then we have a weak difference between sets. In this case (53a) is equivalent to (53b):

(53) a. It is not true that Leo and Lea read the same books.

b. Leo and Lea read different books.

Consequently, sentences of the form (54a) or (54b) have the semantics in (55):

a. NP TV NOT THE-SAME(CN).

b. NP TV DIFFERENT(CN)

$Q^{d} R \cap X \nsubseteq Q R \cap X$

We have seen (cf. examples (19a) and (19b)) that it is preferable to treat, roughly, $N O(X)$ as $\neg \operatorname{SOME}(X)$. The same is true with different. For instance (56a) is equivalent to $(56 b)$ :

(56) a. No (two) students read different books.

b. It is not true that some (two) students read different books.

Given the fact that $\operatorname{SOME}(X)(T W O(X))$ is the dual of $E V E R Y(X)(E V E R Y$ $T W O(X)$ ), the semantics in (55) and in (15) indicates that (57a) and (57b) are equivalent, which seems reasonable (Keenan 2005):

(57) a. Every two students read different books. 
Semantics of the same

b. No two students read the same books.

The above equivalence can be treated as an argument for considering different to involve the weak difference between sets. If it appears that in some cases the strong difference is necessary (to treat some modifications of different, like for instance completely different) then there are at least two possibilities. Thus what (53b) means (under the logical reading with strong difference between sets) is that either Leo or Lea read a book and no book which was read by Lea or Leo was read by both of them. This observation generalised in the same way as the one which gave rise to (15) gives (58a) as the semantics of sentences of the form in (54b). The second possibility of a "strong reading" is given in (58b). I will not comment these two possibilities:
a. $Q^{d} \operatorname{SOME}(X)_{a c c} R \wedge Q^{d} R \cap X \subset \neg Q R \cap X$
b. $Q^{d} \operatorname{SOME}(X)_{a c c} R \wedge Q R \cap X=\emptyset$

The semantics of different occurring in sentences of other than standard types can easily be given. For instance the conjunctive sentence in (59) has the "weak" semantics in (60a) and the "strong" semantics in (60b):

$N P T N P_{1} A N D T V P_{2}$ DIFFERENT $(C N)$
a. $Q R_{1} \cap X \neq Q R_{2} \cap X$
b. $Q R_{1} \cap Q R_{2} \cap X=\emptyset$

Similarly with other types of sentences.

\section{Categorial status of the same}

The variety of examples discussed above indicates that the syntactic status of the same cannot be simple and shows that it is categorially polyvalent. In this section I will make some remarks related not directly to the syntax of expressions formed with the same but rather to the type of functions denoted by such expressions. I will also indicate some constraints on such functions.

There are three classes of examples which have been discussed above: (1) in simple sentences, the same forms, with a common noun, a verbal argument playing the role of the direct object; (2) in sentences in the comparative form, the same forms a comparative clause; and (3) in sentences with conjoined transitive verb phrases expressing multiple events, the same forms direct-object-like expressions. In all cases the same takes a common noun as one argument. Then in the first and third cases the expressions thus obtained apply to transitive verbs (phrases) to form a verb phrase. In the first type of examples, the verb phrases thus obtained have a special 
status, since in principle they cannot take proper nouns as grammatical subjects. Furthermore, the verbal argument formed in the case of simple examples cannot in principle occur in the subject position. In that respect, they behave, as has been sometimes noticed, like anaphors. Since the same with common nouns forms verbal arguments (which cannot occur in the subject position) it behaves like anaphoric determiners (Zuber 2010b, 2011a).

The question one can ask now is: what is the logical type of the result of the function denoted by the same in the case of standard sentences? We know that such sentences do not take proper nouns as subjects (under the sentence-internal reading) and thus the type of objects denoted by the subject NP cannot be $e$, the type corresponding to individuals. We can suppose that it is of the raised type $\langle\langle e, t\rangle, t\rangle$, which, ignoring directionality, corresponds to the category $S /(S / N P)$. Since the same applied to a common noun forms a verbal argument playing the role of direct object, the same $(C N)$ applies to a transitive verb to form a verb phrase. Semantically, according to (15), this verb phrase denotes a set of type $\langle 1\rangle$ quantifiers. Thus, in order to avoid the type mismatch, the verb phrase must be raised to become of the category $S /(S /(S / N P))$.

What is the status of the same and different in standard sentences? As indicated, they form verbal arguments when applied to a common noun. In that sense they are like "ordinary" (unary) determiners. However, verbal arguments formed by the same and different in standard sentences cannot occur in subject position of a sentence. In this respect they are like anaphoric determiners like no ... but himself and most ... including herself (Zuber 2010b) or the Slavic possessive anaphoric determiner svoj (Zuber 2011a). All of these items can be said to belong to the class of generalised determiners, that is expressions which form a verbal argument with a common noun or a generalised determiner phrase (GDP). Probably some comparative determiners, such as more ... than Lea (as found in Leo knows more languages than Lea), also belong to this class (cf. Zuber 2011b).

I will thus accept that there is a class of generalised determiners, that is expressions which form GDPs which can serve as verbal arguments. The question one can ask is whether there are constraints on denotations of generalised determiners similar to constraints on denotations of "ordinary" determiners. I show now that they satisfy a condition of generalised conservativity.

Obviously the classical notion of conservativity does not apply to denotations of generalised determiners. Functions denoted by generalised determiners have sets as arguments. Their second argument is a binary relation. For such functions the following definition of generalised conservativity can be justified (Zuber 2010a,b, 2011a,b):

(61) A function $F$ from sets and binary relations is conservative iff $F(X, R)=$ 
Semantics of the same

$$
F(X,(E \times X) \cap R) \text {, for any } X \subseteq E \text { and } R \text { a binary relation. }
$$

A generalised determiner applies to a common noun and gives a GDP, which applies to an intransitive verb phrase to give a VP. In the case of standard sentences VPs denote a set of type $\langle 1\rangle$ quantifiers. According to (15), the function denoted by the generalised determiner the same (occurring is standard sentences) can be represented as in (62). Similarly, according to (27a), the function denoted by the same modified by a numeral can be represented by (63) :

$$
\begin{aligned}
& F(X, R)=\left\{Q: Q^{d} R \cap X \subseteq Q R \cap X\right\} \\
& G_{n}(X, R)=\{Q:|Q R \cap X|=n\}
\end{aligned}
$$

It is easy to check that both functions in (62) and in (63) are conservative in the sense of (61).

When occurring in comparative sentences the same and differrent are not generalised determiners, but they form such determiners with the complementiser phrase. Thus the same ... as NP and different ... than NP can be considered generalised determiners. When applied to a common noun, they give a GDP which applies to a TVP and gives a VP. This VP denotes a set. For instance the function denoted by the generalised determiner the same ... as $N P_{2}$ in the schema in (28) above denotes the conservative function given in (64):

$F(X, R)=\left\{x: x R \cap X \subseteq Q_{2} R \cap X\right\}$, where $X$ is the denotation of $C N, Q_{2}$ the denotation of $N P_{2}$ and $R$ the denotation of $T V P$.

Finally, the same in conjunctive sentences can be compared to "ordinary" binary ( $n$-ary) determiners taking two predicative arguments (as in More students danced than sang). Thus the same in conjunctive sentences is a generalised determiner which takes a common noun as argument and two (or more) transitive verbs and gives a verb phrase (whose category is $S /(S /(S / N P))$ ). Consequently the same in this case denotes a function which takes a set and two (or more) binary relations as arguments and gives a set of type $\langle 1\rangle$ quantifiers as result. For instance the same in (38) denotes the function in (65) and the same with a numeral as in (40) denotes the function in (66):

$$
\begin{aligned}
& F\left(X, R_{1}, R_{2}\right)=\left\{Q: Q R_{1} \cap X=Q R_{2} \cap X\right\} \\
& G_{n}\left(X, R_{1}, R_{2}\right)=\left\{Q:\left|Q R_{1} \cap Q R_{2} \cap X\right|=n\right\}
\end{aligned}
$$

"Ordinary" binary determiners denote conservative type $\langle 1\langle 1,1\rangle\rangle$ quantifiers (cf. Zuber 2009). Their conservativity can be generalised as follows to functions taking a set and two binary relations as arguments: 
(67) A function $F$ from a set and two binary relations is conservative iff the following holds: $F(X, R, S)=F(X,(E \times X) \cap R,(E \times X) \cap S)$

Functions in (65) and (66) are conservative.

\section{Conclusion}

The fact that formatives like the same or different give rise to expressions which denote non-reducible type $\langle 2\rangle$ quantifiers (Keenan 1992) does not entail that the semantics of sentences with these formatives cannot be provided using tools of GQT as claimed in Barker 2007. What is shown in Keenan 1992 is that difficulties can arise if one treats the application of type $\langle 1\rangle$ quantifiers to a binary relation in the standard way, that is as the semantic counterpart of the operation of forming a VP by applying a object NP to a transitive VP. I have shown that these difficulties disappear if the type $\langle 1\rangle$ quantifier applies to a binary relation as the semantic counterpart of the subject NP, that is as the nominative extension of a type $\langle 1\rangle$ quantifier. The use of such extensions and of the dual of quantifiers allows us to deal with various logical aspects of the semantics of many structurally different constructions with the same and different.

\section{References}

Barker, Chris. 2007. Parasitic scope. Linguistics and Philosophy 30. 407-444.

Carlson, Greg N. 1987. Same and different: some consequences for syntax and semantics. Linguistics and Philosophy 10(4). 531-565.

Keenan, Edward L. 1992. Beyond the Frege boundary. Linguistics and Philosophy 15. 199-221.

Keenan, Edward L. 2005. Excursion in natural logic. In Claudia Casadio, Philip J. Scott \& Robert A.G. Seely (eds.), Language and grammar: Studies in mathematical linguistics and natural language, 31-52. Stanford: CSLI.

Lasersohn, Peter N. 2000. Same, models and representation. In B. Jackson \& T. Mathews (eds.), Semantics and Linguistic Theory (salt) 10, 83-97.

Zuber, Richard. 2009. A semantic constraint on binary determiners. Linguistics and Philosophy 32. 95-114.

Zuber, Richard. 2010a. Generalising conservativity. In Anuj Dawar \& Ruy de Queiroz (eds.), Logic, Language, Information and Computation 17, 247-258. Berlin and Heidelberg: Springer Verlag.

Zuber, Richard. 2010b. Semantic constraints on anaphoric determiners. Research on Language and Computation 8. 255-271.

Zuber, Richard. 2011a. Semantics of Slavic anaphoric possessive determiners. In 
Semantics of the same

Edward Cormany \& Satoshi Ito (eds.), Semantics and Linguistic Theory (SALT) 19, 464-477. Ithaca, NY: CLC.

Zuber, Richard. 2011b. Some generalised comparative determiners. In Sylvain Pogodalla \& Jean-Philippe Prost (eds.), Logical Aspects of Computational Linguistics 2011, 267-281. Berlin and Heidelberg: Springer Verlag.

Richard Zuber

UMR 7110

CNRS-Université Paris 7

Case 7031

2 place Jussieu

75251 Paris cedex 05

richard.zuber@linguist.jussieu.fr 